2P Y Chiou, ${ }^{3} \mathrm{C} C \mathrm{C}$ Wang, ${ }^{4} \mathrm{P}$ Chuang, ${ }^{3} \mathrm{M}$ Y Yen, ${ }^{1} \mathrm{C}$ L Chang. 'Mackay Medicine, Nursing and Management College, Taipei, Taiwan; ${ }^{2}$ National Yang Ming University, Department of Nursing, Taipei, Taiwan; ${ }^{3}$ Taipei City Hospital, KunMing Branch, Taipei, Taiwan; ${ }^{4}$ Administration Center, Taipei City Hospital, KunMing Branch, Taipei, Taiwan

Purpose This study investigated the difference of effects between advanced partner notification (APN) and traditional partner notification (TPN).

Methods The subjects who had Western bolt test or newly diagnosed with HIV were recruited. All subjects were randomly assigned into experimental and control group, 30 subjects in each group. Advanced Partner Notification was therefore developed based on the self-efficacy concept of Bandura. The process of APN includes advanced interaction model, comprehensive assessment model for partner information, and promoting self-efficacy of partner notification model. In control group, 30 subjects accepted the process of TPN.

Results Sixty participants were men who have sex with men and unmarried. The mean age was 28.3 years $(S D=4.64)$. The results revealed that the index cases of APN were significantly better than the group of TPNM in provided more contactable partner of 107 cases $(t=2.16, p=0.037)$, successed notified more partner of 73 cases $(t=2.25, p=0.029)$, receiving HIV test more partner of 25 cases $(t=2.05,=0.046)$. There were 22 partners whose HIV test were positive in APN group (HIV positive rate was 41.51\%) and 7 partners whose HIV test were positive in TPN group (HIV positive rate was $25.0 \%$ ). The HIV positive partners in APN group were 15 cases $(t=2.64, p=0.01)$ more than those in TPN group. In addition, the mean difference in safer sexual knowledge, number of sexual partners, frequency of unsafe sexual behaviours, frequency of safer sexual behaviours, frequency of resource referral numbers, and process evaluation of PN were significantly better than those in TPN group.

Conclusion The process of APN is better than the process of TPN in many aspects. The result can improve the quality of current partner notification policy and practise.

\section{P5.002 PARTNER NOTIFICATION OUTCOMES FOR MSM AND HETEROSEXUALS WITH STI/HIV: CHALLENGES AT DIFFERENT STAGES}

doi:10.1136/sextrans-2013-051184.1049

${ }^{1} \mathbf{F}$ van Aar, ${ }^{1} Y$ van Weert, ${ }^{2} \mathrm{R}$ Spijker, ${ }^{3} \mathrm{H}$ Götz, ${ }^{1} \mathrm{E}$ Op de Coul, Partner Notification group. 'National Institute for Public Health and the Environment, Bilthoven, The Netherlands, ${ }^{2}$ STI AIDS The Netherlands, Amsterdam, The Netherlands; ${ }^{3}$ Municipal Public Health Service Rotterdam-Rijnmond, Rotterdam, The Netherlands

Background Partner notification (PN) is seen as a vital tool to break HIV/STI transmission chains. In the Netherlands, studies assessing PN effectiveness were lacking. Here, we evaluated effectiveness of current PN practises in STI clinics to provide recommendations to further enhance PN.

Methods PN outcomes were collected through a newly developed registration system from index patients with HIV, syphilis, and gonorrhoea visiting five STI centres in 2010-2011. PN outcomes for men who have sex with men (MSM) and heterosexuals included partners: at risk, notifiable, notified, tested and diagnosed with STI/HIV.

Results Of all index patients newly diagnosed with HIV/STI $(\mathrm{N}=388)$ for whom PN was indicated, $312 \mathrm{MSM}$, 35 heterosexual men and 41 women reported respectively 2042, 126 and 82 partners at risk (6.5, 3.6 and 2.0 partners per index). Proportions of notifiable partners differed significantly by sexual preference (MSM: 46\%, heterosexual men: $63 \%$, women: $87 \%, p<0.001)$. Proportions of notified partners (of those notifiable) were lowest for heterosexual men ( $77 \%$ versus $92 \%$ for MSM and $83 \%$ for women, $p<0.001$ ). STI positivity rates among partners were high for all groups: $33 \%-50 \%$.
Conclusions The major challenge in PN among MSM remains the large proportion of unnotifiable (anonymous) partners, whereas among heterosexuals a higher percentage partners were lost during the actual notification process. Therefore, newly developed interventions, e.g.anonymous internet-based $\mathrm{PN}$ or $\mathrm{PN}$ training, should be tailored to specific risk-groups.

\section{P5.003 ACCEPTANCE OF AN ONLINE PARTNER NOTIFICATION TOOL FOR STI, CALLED SUGGEST-A-TEST}

doi:10.1136/sextrans-2013-051184.1050

${ }^{1}$ M S van Rooijen, ${ }^{2} \mathrm{P}$ Vriens, ${ }^{2,3} \mathrm{H}$ Gotz, ${ }^{1} \mathrm{~T}$ Heijman, ${ }^{2,3} \mathrm{H}$ Voeten, ${ }^{1} \mathrm{R}$ Koekenbier. ${ }^{1}$ Cluster of Infectious Disease, Municipal Public Health Service Amsterdam, Amsterdam, The Netherlands; ${ }^{2}$ Department Infectious Disease Control, Public Health Service RotterdamRijnmond, Rotterdam, The Netherlands; '3epartment of Public Health, Erasmus MC, Rotterdam, The Netherlands

Background Partner notification (PN) is effective in early detection and treatment of STI. After counselling internet-based PN might be an additional tool in stimulating STI positive clients to notify partners. In this study we evaluate the acceptance of an internet-based application, Suggestatest.nl (SAT).

Methods To notify, an index has to login using a nurse-generated code. Notifications to the partner can be sent by email, text message, postal letter or gay dating site. The partner receives a message with a unique code and has to login on the site to see the notified infection. Based on this message including code, the client is tested and treated at the STI clinic.

SAT was piloted in STI clinics in Rotterdam and Amsterdam, the Netherlands. Questionnaire data were collected online for index clients and on paper at the STI clinics for partners notified through SAT.

Results In 8 months 55 index clients completed the questionnaire (16.9\%). Using internet for PN is experienced as confidential and safe by $67 \%$ (37/55); $87.3 \%$ (48/55) of clients find online PN easy. $85 \%(47 / 55)$ find SAT an acceptable tool to notify for STI, compared to only $45 \%(25 / 55)$ for HIV. $82 \%(45 / 55)$ would like being notified themselves by SAT.

43 of the 1,123 partners who were notified by SAT completed a questionnaire (3.8\%) upon presenting at the clinic. 91\% (39/43) find it useful and $88 \%(38 / 43)$ find it acceptable that the STI clinic offers SAT. $88 \%(38 / 43)$ and $53 \%$ (23/43) find it acceptable to use SAT to notify for STI and HIV, respectively.

Discussion These preliminary data show that index patients and partners find it acceptable to use SAT to notify for STI, but they are less favourable to use SAT to notify for HIV.

Data collection is still ongoing; updated data will be presented at the ISSTDR.

\section{P5.004 INITIAL UPTAKE OF STI PARTNER NOTIFICATION WEBSITE SO THEY CAN KNOW}

doi:10.1136/sextrans-2013-051184.1051

1J H Ladd, ${ }^{1} \mathrm{~J}$ McManus, ${ }^{2} \mathrm{M}$ Rushanan, ${ }^{3} \mathrm{~L}$ Barclay, ${ }^{4} \mathrm{~S}$ Adelson, ${ }^{1,5} \mathrm{C}$ Gaydos. ${ }^{1}$ Sexual Health Innovations, San Francisco, CA, United States; ${ }^{2}$ Information Security Institute, Johns Hopkins University, Baltimore, MD, United States; ${ }^{3}$ American Sexual Health Association, Research Triangle Park, NC, United States; ${ }^{4}$ Adelson Consulting, Chelsea, MA, United States; ${ }^{5}$ Division of Infectious Diseases, Johns Hopkins University, Baltimore, MD, United States

Background So They Can Know (STCK) is a partner notification website developed from extensive formative research conducted with the target population and health care providers. STCK allows patients in the United States to send informative anonymous notification emails for curable STIs to their partners. STCK captures selfreported reasons for sending emails, a unique aspect of the website. 